\title{
A Meteorological Window Forecast System for Immersed Tunnel Construction
}

\author{
Lin Meihong $^{(1)}$, Lü Weiqing ${ }^{(2)}$, Ying Zongquan ${ }^{(1)}$, Su Linwang ${ }^{(1)}$ \\ ${ }^{(1)}$ CCCC Fourth Harbor Engineering Institute Co., Ltd., Guangzhou, China 510230 \\ ${ }^{(2)}$ CCCC Fourth Harbor Engineering Co., Ltd., Guangzhou, China 510231 \\ lmeihong@gzpcc.com, lvweiqing@gzpcc.com, yzongquan@gzpcc.com, slinwang@gzpcc.com
}

\begin{abstract}
This paper presents a solution for force and stability meteorological window forecast system of large-scale tunnel elements based on $\mathrm{C} / \mathrm{S}+\mathrm{B} / \mathrm{S}$ mode, through the plug-in framework design pattern, the strong coupling relationship of each subsystem can be reduced in the process of system integration; the massive environmental data is stored by open source data warehouse technology, and storage solution with fast statistical analysis support is developed; based on superiority method and satisfaction value method, multiattributes group decision making algorithm for force and stability window condition screening and window forecast algorithm is also provided, these methods can take a quick solution for meteorological window limit conditions. The system is applied to force and stability meteorological window forecast for the construction process of tunnel elements, the test results show that the system has a good portability, versatility and scalability.
\end{abstract}

Keywords-meteorological window forecast; open source data warehouse technology; multi-attributes group decision making; superiority metho; satisfaction value method

\section{INTRODUCTION}

As an important cross-realm transportation infrastructure type, because of little influence of shipping, immersed tunnel is considered to be the first choice for busy shipping across the waters. But bad construction condition has great influence in the process of tunnel construction, in order to meet the requirements for towing, mooring, sinking construction of tunnel elements, stable environmental conditions are need to screening, and essential safety measures must be planed for sudden events. With the consideration of large investment, construction time requirements, decision making analysis is very necessary. Considering hydro meteorological environmental conditions and characteristics of immersed tunnel, making correct construction decision is the key problem.

Decision support system is widely applied in ship scheduling control ${ }^{[1]}$, freight transportation, container allocation, dams and highway bridge construction ${ }^{[2]}$. With the consideration of environmental complexity, typhoon influence, long operating cycle, it needs to take more judgment parameters for the analysis of force and stability. This paper developed a decision making support system ${ }^{[3]}$ based on the force and stability analysis database. By means of multi-attributes decision making advantage method, satisfaction value method ${ }^{[4,5]}$, the system provides analysis basis for environmental condition judgment of force and stability. Finally, the system is applied to force and stability meteorology window forecast analysis and gives decision making for tunnel construction support.

\section{SYSTEM IMPLEMENTATION}

\section{A. System architecture}

The system was composed of several subsystems, mainly includes basic data management, force and stability calculation analysis, meteorology window forecast and web publish. The basic data management subsystem includes environmental data management and engineering construction data management modules; Force and stability calculation analysis subsystem includes stability analysis, towing transportation analysis, mooring system analysis and sinking construction analysis modules; Meteorology window forecast subsystem includes engineering empirical data and meteorology window forecast modules; web publish subsystem includes basic data publish, force and stability data publish and meteorology window data publish modules. Because of generalized, multi-source, heterogeneous of system dependency, the system is designed by using the four layer model: display layer, business layer, persistence layer and store layer. The system general structure is shown in fig.1.

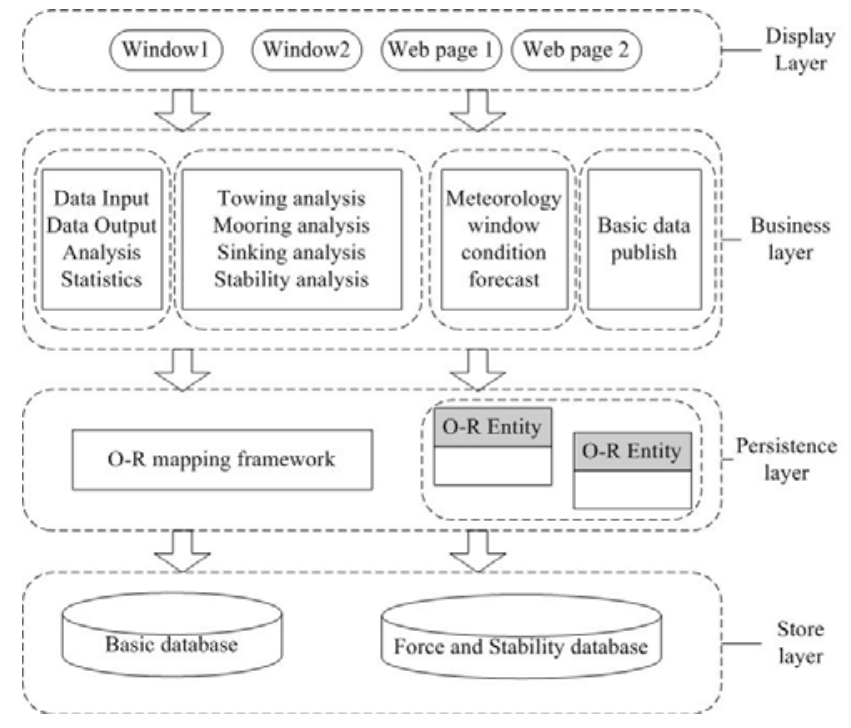

Figure 1. System architecture 


\section{B. System functions}

Considering construction characteristics of over-water concrete precast element, this paper develops the force and stability meteorology window forecast system, which includes subsystems: basic data management, force and stability calculation analysis, meteorology window forecast and web publish. Finally, application of practical project demonstrates the system's feasibility. The functions of each subsystem are as follow:

\section{1) Basic data management subsystem}

With the establishment of the file data and database data, which includes environmental data, geometry data of tunnel elements, force and stability data and mooring system data etc, combined with the environmental monitoring data, the subsystem provides such functions: the storage of basic data, input/output and query analysis of basic data, these functions provide data source and analysis basis for other subsystems.

2) Force and stability calculation analysis subsystem

On the basis of basic data subsystem, the calculation analysis file is generated, and the force and stability can be analysed by standard, empirical formula and ANSYS AQWA program. This subsystem implements force analysis for tunnel element under the comprehensive action of environmental load and working load. By mean of the generation of ANSYS AQWA parameter input file for each possible calculation cases, and the extraction of force and stability results, the subsystem can establish the force and stability database.

3) Construction operation meteorology window forecast subsystem

According to the calculation results of force and stability database, with the input of force and stability limiting condition, the window forecast screening operation can be executed on the force and stability database, and the wind, wave and current limiting condition can be established, with the processing of environmental monitoring data, finally, the meteorology window data can be screened by search algorithm.

4) Web publish subsystem

By means of web technology, geographic information, monitoring and prediction data of marine environment, force and stability analysis data of tunnel elements and construction operation meteorology window forecast data can be published, and project management personnel can inquire conveniently.

\section{KEY TECHNOLOGIES}

\section{A. Application integration based on plug-in framework}

The whole system includes the basic data subsystem, force and stability analysis subsystem and construction operation window forecast subsystem. All the data of the system is encapsulated by the data layer, and the integrated management of interface views is the key problem. In the conventional method, it needs to define various types of interface of each subsystem, it usually takes more modification work based on each system, and the whole application framework always is a strong coupling of each subsystem. In order to reduce coupling and definitions of call interface, based on the adapter method and protocol definition method, the system is designed, the views of each subsystem are packaged by the subsystem adapter, and the subsystem adapter can be integrated by the main system adapter through the interface protocol of subsystems, finally, the views can be integrated under the main program.

\section{$B$. The design and storage of environmental data}

Environmental data is the core of the decision support, it includes the history of environmental(wind, wave and current) time data , forecast time data and monitoring data. Parameter objects of each type include spatial data, time characteristics and proprietary data attributes. Analysis can be taken by means of object-oriented technology, and the mapping between data storage and data object can be implemented. In the implementation of environmental data modeling, the process of data modeling needs to consider the following factors:

1) the same spatial coordinate system;

2) Three-dimensional, time-varying and multi-scale features;

3) Stratification, combination, regional characteristics;

4) The whole environmental information systems include time and spatial data;

5) Quickly import and export support; upon import, without modifying;

6) Query statistical analysis of the massive data support.

The implementation of environmental data objectoriented modeling, according to the characteristics of its hierarchy, combinations, timing, spatial, abstract classes of data model can be defined: abstract classes of environmental parameters, wind parameters, wave parameters, location grid and other hydrological environment parameters etc. Abstract class of environmental parameters includes: Parameter ID, parameter code, type, group ID, hierarchical ID, time data, hierarchy data and space environmental data. Other parameter classes contain the proprietary nature data. The structure is shown in fig. 2.

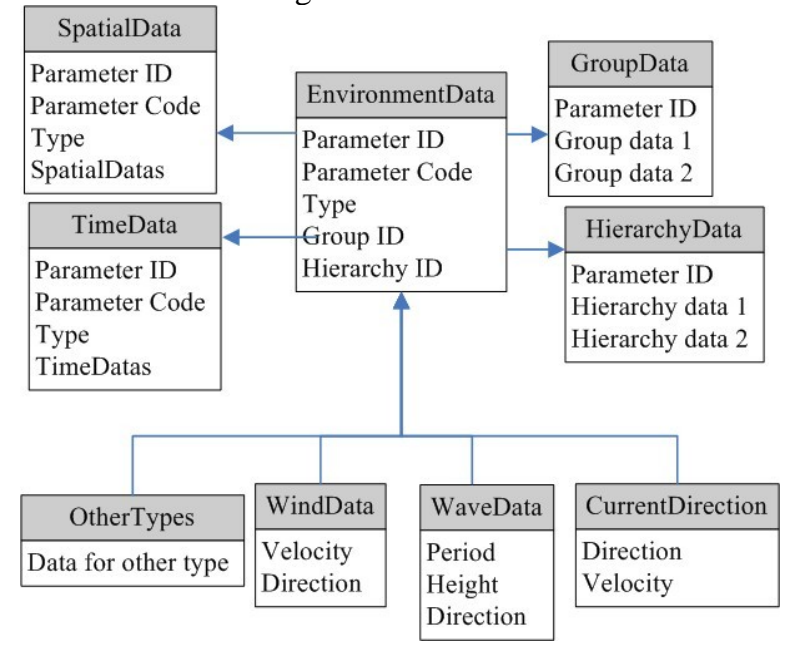

Figure 2. System architecture 


\section{Meteorology limit conditions screening algorithm} based on multi-attributes group decision-making method

The analysis decisions set of tunnel element construction process, each decision includes conditions and results. The conditions include wind speed, wind direction; wave period, wave height, wave direction; current velocity, current direction. The results include environmental load results, motion response results, mooring and external load calculation results and other external forces calculation results, etc. Force and stability judgment conditions mainly aimed at the force and motion response results, the process of decision support is: according to the judgment conditions, the decision making set is screened to obtain optimal environmental limit conditions. According to the results of optimal environmental limit conditions, construction support can be provided and statistical analysis for meteorological data can be taken based on the optimal environmental limit conditions, finally the construction window can be determined. There are several main analysis points:

1) To consider the parameters: wind speed, wind direction; wave height, wave direction, wave period; current velocity, current directions;

2) Decision making set must contain force and stability calculation results that may be encountered in the whole construction processes.

3) According to a particular force and stability judgment condition, arbitrary two scheme, there exists mutual independence (can not eliminate at the same time) or strict preference relation (can eliminate one).

4) For a given excision value, when the corresponding parameter value of the scheme is below, above, not equal or equal, the scheme can be eliminate from the whole set.

The above $1 \sim 2$ is the precondition of the decision making set, $3 \sim 4$ is the description for superiority method and satisfaction value method for multi-attributes screening. According to the analysis of the force and stability conditions, decision makers of different conditions is simulated, superiority matrix and satisfaction value matrix for multiple decision makers can be established, finally, the decision making set can be screened according to these matrices. The specific algorithm is as follows:

1) Define decision making set $A=\left\{A_{1}, A_{2}, \cdots, A_{n}\right\}$, which is created by the fore and stability results;

2) Define satisfaction value decision makers by each satisfaction value parameter, define satisfaction value matrix $C_{m n}$ (m:the count of decision makers, $\boldsymbol{n}$ : the count of decision making set), the initial value of $C_{m n}$ are set to 0 , for decision condition $\boldsymbol{i}$, if decision scheme $\boldsymbol{j}$ can be eliminated according to decision maker $i$, then $C_{i j}=1$, then, define $S_{n}=\sum_{i=1}^{m} c_{i n}$, finally, create a new set which includes decision scheme of $A=\left\{A_{1}, A_{2}, \cdots, A_{n}\right\}$ by the condition: $S_{i}=0$;
3) According to the new set from (2), for each environmental condition of each decision scheme, define a decision maker, define superiority matrix $P_{n n}$ (m:the count of decision makers, $n$ : the count of decision making set), the initial value of $P_{n n}$ are set to 0 , for decision condition $\boldsymbol{i}$, if decision scheme $\boldsymbol{j}$ can be eliminated according to superiority decision maker $\boldsymbol{i}$, then $P_{i j}=1$, then, define $S_{n}=\sum_{i=1}^{m} P_{i n}$, finally, create a new set which includes decision scheme of $A=\left\{A_{1}, A_{2}, \cdots, A_{n}\right\}$ by the condition: $S_{i}=0$;Finally, the decision set can be established, two different decision making sets can be merged by the above algorithm.

D. meteorology window forecast algorithm based on multiattributes decision-making superiority method

According to the decision making set, environmental parameters can be extracted by each decision scheme, for given environmental (wind, wave and current) time history data, the algorithm is put forward to judge the time of the data point can be use to update the time range. The key point is that: for each data point, the decision-making set has a priority sequence, and there is a optimal decision scheme, the time of data point can use to update the total time range or not by comparison with the optimal decision scheme.

The algorithm is based on weight value determination method, for a given data point $U_{i j}$, different weights $w_{j}$ are considered for wind direction, wave direction and current direction, each $w_{j}$ indicates the degree of importance, and the weight of other parameters are set to zero. For each decision scheme, compute the total weight: $S_{i}=\sum_{j=1}^{m} w_{j}\left(U_{i j}-u_{i j}\right)$, where $u_{i j}$ is the elements of limit conditions, the scheme which has the minimum value is the target scheme.

\section{APPLICATION OF FORCE AND STABILITY METEOROLOGY WINDOW FORECAST SYSTEM}

The system is established based on the decision making set of force and stability results, according to the judging condition of towing resistance, mooring force and motion response parameters, the system can screening the decision making set to obtain optimal environmental limit conditions. The screening parameters can be appointed dynamically, this gives engineers and technicians a way for multi-parameters screening.

The environmental condition use for analysis is: current direction: SSW, S, current velocity: $0.5 \sim 1.1 \mathrm{~m} / \mathrm{s}$; wave height: 0.5 1.0m, wave period: 5 7s, wave direction: NNE, N, NNW, SSW, S, SSE; wind: 6 grade, direction: NNE, N, NNW, SSW, S, SSE. Force and stability results are 
reconstructed and saved to database, and formed the decision making set.

The mooring scheme in showed in fig.3. Assume the environmental limit conditions: current velocity is $1.0 \mathrm{~m} / \mathrm{s}$, current direction S; wave height $0.8 \mathrm{~m}$; and force and stability condition: the ratio(safety factor) of maximum mooring line force and average mooring line force can not exceed 1.5. according to the environmental limit conditions, we can obtain decision making set. Fig. 4 shows the safety factor of wave period 5s,6s,7s, and finally the wave period limit conditions can be screened and showed as fig.5. In the same way, we can obtain all the meteorology window limit conditions for current velocity $1.0 \mathrm{~m} / \mathrm{s}, 1.1 \mathrm{~m} / \mathrm{s}$, wave height $0.8 \mathrm{~m}$ :

1) Current velocity $1.0 \mathrm{~m} / \mathrm{s}$ :

When current is $1.0 \mathrm{~m} / \mathrm{s}$, wave height is $0.8 \mathrm{~m}$, the wave period should not exceed $6 \mathrm{~s}$, but when the wave direction is NNW, NNE, SSW and SSE, the period should not exceed $5 \mathrm{~s}$.

\section{2) Current velocity $1.1 \mathrm{~m} / \mathrm{s}$}

When current is $1.1 \mathrm{~m} / \mathrm{s}$, wave height is $0.8 \mathrm{~m}$, the wave period should not exceed 5 s except wave direction: $\mathrm{N}$, S. When wave direction is $\mathrm{N}, \mathrm{S}$ the period should not exceed $6 \mathrm{~s}$.

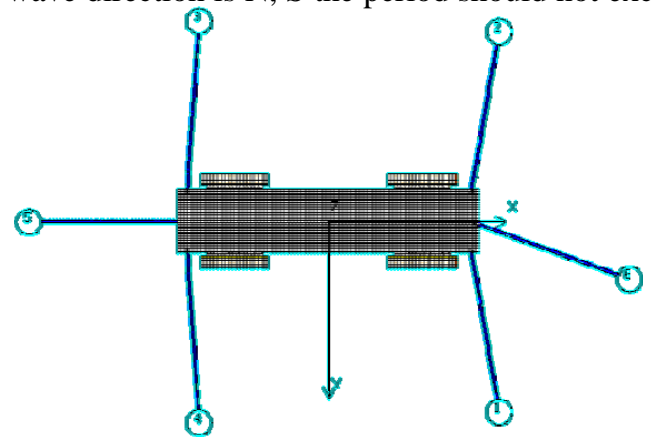

Figure 3. Mooring scheme

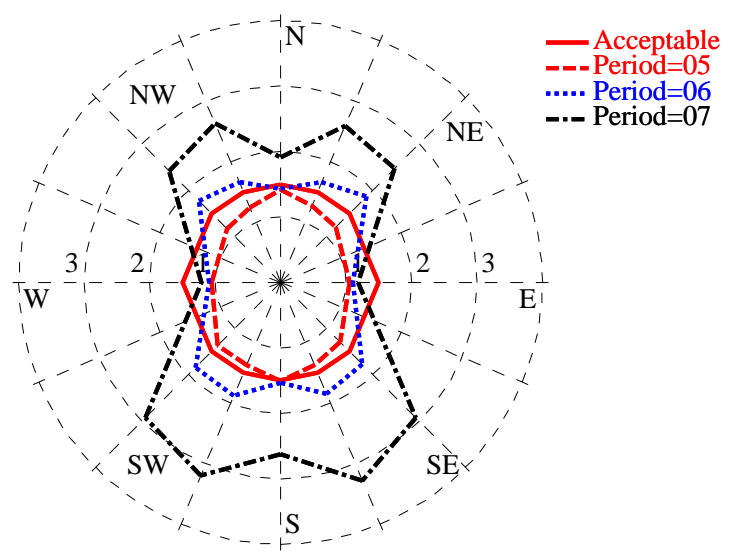

Figure 4. Mooring force ratio of period $5 \mathrm{~s}, 6 \mathrm{~s}, 7 \mathrm{~s}$

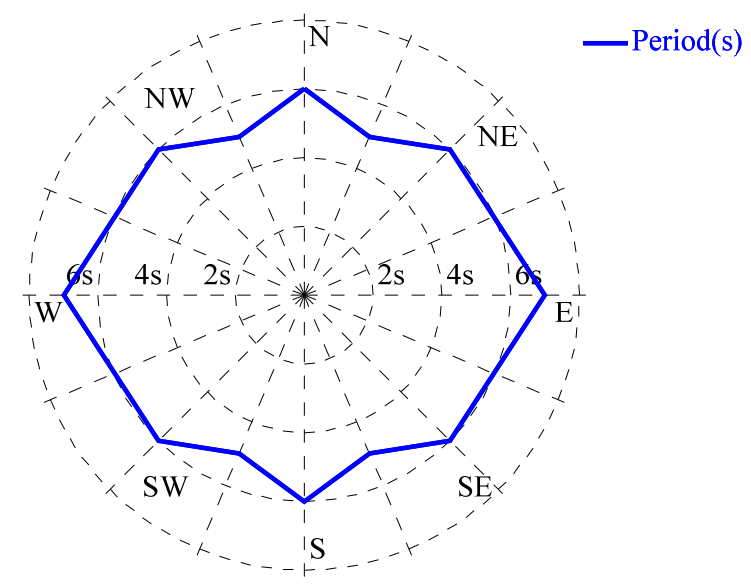

Figure 5. Wave period window limit condition of current velocity:1.0m/s

\section{CONCLUSION}

This paper provides a solution for force and stability meteorology window forecast system of tunnel elements, its suggested that through the establishment of decision support database and multi-attributes group decision making algorithm, the meteorology window forecast problem for multi-parameters can be solve clearly, and the algorithm provides environmental limit condition results for meteorology window forecast.

1) The complexity of environmental database can be redcuced by fully model design, and the demard for environmental data storage and analysis can be solved.

2) The completeness and accuracy of force and stability decision making database is the key to the subsequent analysis, multi-attributes decision-making method provides a better way for analysis, but research remains to be done in terms of efficiency.

3) This paper gives the analysis methods for force and stability environmental limit conditions, which mainly built on the basis of decision making set, but less work to do with influence law of force and stability results. for that, the data mining research remains to be done.

\section{REFERENCES}

[1] LIU Yuhong, "The structure design of an intelligent decision support system for navigation collision avoidance," Proceedings of the 3rd International Conference on Machine Learning and Cybernetics, 2005: 3997-4003.

[2] Andersen E. Y., Pedersen L, "Structural monitoring of the Great BeltEast Bridge,” Strait crossings, 1994; Rotterdam: Balkema: 189195.

[3] DeSanctis G, Gallupe R B, “A Foundation for the study of group decision support systems,” Management Science, 1987,33(5):589-608.

[4] Pawlak Z, "Rough sets," International Journal of Computer Information Sciences, 1982, 11(5):342-356.

[5] Pawlak Z, "Rough sets: Theoretical aspects of reasoning about data," Dordrecht: Kluwer Academic Publishers, 1991 